Chirurg 2013 $\cdot 84: 434$

DOI 10.1007/s00104-013-2505-0

Online publiziert: 12. April 2013

(c) Springer-Verlag Berlin Heidelberg 2013
T. Franzke $\cdot$ J. Jähne

Klinik für Allgemein- und Viszeralchirurgie, Schwerpunkt für endokrine und onkologische Chirurgie,

Diakoniekrankenhaus Henriettenstiftung, Hannover

\title{
Risikofaktoren für ein peritoneales Rezidiv bei ösophagogastralen Siegelringkarzinomen
}

\section{Ergebnisse einer retrospektiven Multicenterstudie}

\section{Ergebnisse}

Von insgesamt 3010 Patienten hatten 1050 $(34,9 \%)$ ein Siegelringkarzinom. $424 \mathrm{~Pa}-$ tienten $(14,1 \%)$ erfüllten die Einschlusskriterien, wobei in $68,9 \%$ der Fälle ein Magenkarzinom vorlag. Mit 51,4\% war die Gastrektomie die am häufigsten durchgeführte Operation. 16,7\% $(\mathrm{n}=71)$ der Patienten wurden neoadjuvant chemotherapiert ( $2 \%$ neoadjuvante Radiochemotherapie $[\mathrm{RCT}])$. Eine adjuvante Behandlung erhielten 35,4\% der Patienten ( $\mathrm{n}=150 / 61 \%$ CTX/38\% RCT/1\% Radiatio). Die histologische Auswertung zeigte 204 Patienten $(49,7 \%)$ mit einem lokal fortgeschrittenen Befund (pT3-4), davon waren 72,9\% nodal-positiv. Bei einem medianen Followup von 54 Monaten kam es bei 50,5\% der Patienten $(n=214)$ zu einem Rezidiv, welches bei $19,1 \%(n=81)$ peritoneal lokalisiert war. Die multivariate Analyse zeigte 4 unabhängige, mit einem peritonealen Rezidiv assoziierte Risikofaktoren: eine Linitis plastica, die Tumorausbreitung bis in die peritoneale Serosa, tumorpositive Lymphknoten sowie die Tumorlokalisation im Magen. Das mediane Überleben nach der initial kurativen Resektion lag bei 17,7 Monaten mit einer 1-/3- und 5-Jahres-Überlebensrate(-JÜR) von 69,2, 18,1 und 5,9\%.

Die zwischen 1997 und 2010 erhobenen Daten aus 19 französischen Tumorzentren wurden retrospektiv ausgewertet. In die Studie wurden nur nichtfernmetastasierte R0-resezierte Patienten eingeschlossen.

\section{Diskussion}

Mehrere Studien konnten den signifikanten Einfluss eines peritonealen Rezidivs auf das Überleben von Patienten mit einem Adenokarzinom des Magens sowie des Ösophagus zeigen. Der aktuelle
Standard einer neoadjuvanten Therapie bei fortgeschrittenen Tumorstadien erbrachte, wie auch eine adjuvante Therapie, in der Subgruppe der Siegelringkarzinome keinen Benefit. Hingegen zeigten erste Ergebnisse für die Durchführung einer prophylaktischen HIPEC eine signifikante Zunahme der 5-JÜR. Die Autoren empfehlen daher ein adaptiertes Vorgehen unter Zuhilfenahme der evaluierten Risikofaktoren mit optionaler Durchführung einer prophylaktischen HIPEC.

Die vorliegende Studie weist erhebliche methodische Mängel auf. Zum einen wird nicht eindeutig zwischen Magenund Ösophaguskarzinomen unterschieden und zum anderen ist die Studienpopulation sehr heterogen. Durch die inhomogenen Therapiekonzepte kann das peritoneale Rezidivrisiko nicht wirklich erfasst werden, sodass auch die ausgesprochen Empfehlungen, trotz aller theoretischen Vorteile einer prophylaktischen HIPEC, zunächst zurückhaltend betrachtet werden sollte.

\section{Korrespondenzadresse}

\section{Dr. T. Franzke}

Klinik für Allgemein- und Viszeralchirurgie, Schwerpunkt für endokrine und onkologische Chirurgie, Diakoniekrankenhaus Henriettenstiftung, Marienstr. 72-90, 30171 Hannover Timm.Franzke@ddh-gruppe.de

Interessenkonflikt. Der korrespondierende Autor gibt für sich und seinen Koautor an, dass kein Interessenkonflikt besteht. 